\title{
Suboptimal Pneumococcal Pneumonia Vaccination Rates Among Patients at Risk in a Managed Care Organization in Israel
}

\author{
NATAN R. KAHAN, RPh, MHA; DAN-ANDREI WAITMAN, MD, MPH; \\ SHIMON BLACKMAN, BScPharm; and DAVID P. CHINITZ, PhD
}

\begin{abstract}
OBJECTIVE: Pneumococcal pneumonia is a vaccine-preventable disease that poses a significant threat to immunocompromised patients. Vaccination rates tend to be low despite recommendations for vaccination in several groups of high-risk patients including any person aged 65 years or older. The purpose of this study was to (a) evaluate the vaccination rates among high-risk patients in a managed care setting in Israel and (b) gain a better understanding of the factors associated with suboptimal use of this vaccine.
\end{abstract}

METHODS: Data were extracted from the electronic medical records of the managed care organization for patients with dates of service from January 2000 to December 2004 for whom the vaccine is recommended. Patients were identified via diagnosis codes according to the International Classification of Diseases, Ninth Revision, Clinical Modification (ICD-9-CM). Vaccination rates were calculated for patients in each disease category. These high-risk patients were contacted to participate in a telephone survey to evaluate the variance in knowledge and awareness levels of the disease between the vaccinated and unvaccinated patients.

RESULTS: A total of 672 patients were identified by the ICD-9-CM codes; 140 $(20.8 \%)$ had been vaccinated and $532(79.2 \%)$ were unvaccinated. Vaccination rates were highest among patients with solid organ transplants $(33.3 \%)$, followed by nephrotic syndrome $(29.4 \%)$, bone marrow transplants $(10.2 \%)$, and human immunodeficiency virus (HIV, 9\%), for an overall rate of $20.8 \%$. Of these patients, survey responses were obtained from 364 (54.2\%). Respondents who were unvaccinated tended to be less well informed about which patient populations are at risk for the disease and the availability of the vaccine.

CONCLUSION: The pneumococcal vaccination rate among immunocompromised patients in this managed care organization was found to be inadequate, at just $20.8 \%$ of the target population. Approaches based on direct contact with the patient, such as by a case manager, may be more successful in the future.

KEYWORDS: Pneumococcal pneumonia vaccine, Vaccination rates, Managed care, Insular populations, Immunocompromised

J Manag Care Pharm. 2006;12(2):152-56

\section{Authors}

NATAN R. KAHAN, RPh, MHA, is a pharmacoepidemiologist, Leumit Health Fund, Tel Aviv, Israel, and a doctoral candidate in the Hadassah Medical Organization School of Public Health, The Hebrew University of Jerusalem, Israel; DAN-ANDREI WAITMAN, MD, MPH, is chairman, Medicines Committee, and SHIMON BLACKMAN, BScPharm, is a clinical pharmacist, Leumit Health Fund; DAVID P. CHINITZ, PhD, is a senior lecturer in health policy, The Hebrew University of Jerusalem.

AUTHOR CORRESPONDENCE: Natan R. Kahan RPh, MHA, Pharmacoepidemiologist, Leumit Health Fund, 23 Schprintsak St., Tel Aviv 64738, Israel. Tel: 972-3-697-0349; Fax: 972-3-696-3589; E-mail:nkahan@leumit.co.il

Copyright $\subset$ 2006, Academy of Managed Care Pharmacy. All rights reserved.
$\mathrm{P}$ neumococcal disease, particularly pneumonia, bacteremia, and meningitis, is a vaccine-preventable disease that is an important cause of mortality and morbidity, especially in vulnerable groups such as the elderly, very young, patients with chronic disease, and the immunocompromised. ${ }^{1}$ High-risk groups include persons with human immunodeficiency virus $(\mathrm{HIV})^{2}$; patients with chronic renal insufficiency, including nephrotic syndrome $e^{3,4}$; individuals with anatomic asplenia ${ }^{5,6}$; and solid organ transplant recipients being treated with immunosuppressive drugs. ${ }^{7.8}$ The vaccine has been recommended for anyone aged 65 years and older as well as patients aged 2 years and older who have the following high-risk medical conditions: chronic heart, kidney, or lung disease (except asthma); cirrhosis of the liver; alcoholism; diabetes mellitus; chronic cerebrospinal fluid leak; other diseases that suppress the immune system; and sickle cell disease. ${ }^{9}$

In the United States, pneumococcal disease has been estimated to account for 3,000 cases of meningitis, 50,000 cases of bacteremia, 500,000 cases of pneumonia, and 7 million cases of otitis media annually. Furthermore, it has been estimated that pneumococcal infection causes approximately 40,000 deaths annually in the United States, accounting for more deaths than any other vaccine-preventable bacterial disease. ${ }^{10}$

Efficacy rates of the vaccine in imunocompetent adults for the prevention of pneumococcal bacteremia and meningitis have been reported to be from $65 \%$ to $75 \%$. However, despite this reported efficacy and although pneumococcal vaccination has been recommended for imunocompetent patients with indications for its administration, ${ }^{11}$ vaccination rates in the United States among persons aged 18 to 64 years with one or more risk conditions in 2002 continued to be low (19.1\%). ${ }^{12}$ Furthermore, despite these recommendations, pneumococcal vaccination has only been included as a Health Plan Employer Data and Information Set (HEDIS) performance measure for patients aged $>65$ years. ${ }^{13-15}$ Consequently, managed care organizations (MCOs) are presently lacking a benchmark to monitor vaccine performance rates among patients in risk groups other than older age.

The present study was conducted in the Leumit Health Fund, one of the 4 MCOs in Israel, which provides the government-mandated national health package to approximately 650,000 members throughout the State of Israel, representing about $10 \%$ of the population. In addition to adopting the vaccination policy for pneumococcal vaccine common to other Western countries, Leumit management has targeted high-risk patient populations to receive the vaccine without having to pay 
a copayment. These subpopulations include patients who have undergone solid organ transplants, bone marrow transplants, and splenectomy as well as patients diagnosed with HIV or nephrotic syndrome.

Throughout the years 2000-2004, postcards in Hebrew were sent to all Leumit Health Fund members for whom the vaccine is recommended, who are eligible to receive the vaccine free of charge, and who had not been vaccinated in the previous 5 years. The postcard informed these members that they were eligible to receive the vaccine free of charge at any of the MCO's approximately 300 clinic sites. The purpose of this study was to evaluate the actual vaccination rates among these patients and to gain a better understanding of the factors associated with the suspected suboptimal utilization of this vaccine.

\section{Methods}

Data were extracted from the electronic patient records of the Leumit Health Fund of Israel. All patient records were identified with one or more of the International Classification of Diseases, Ninth Revision, Clinical Modification (ICD-9-CM) diagnosis codes for a condition for which the vaccine is recommended (Table 1). The vaccination rates were calculated for patients in each disease category. Asplenic patients were not included in the study since this group includes patients who underwent emergency splenectomy and were then vaccinated in the hospital.

A telephone survey was conducted among these patients, both vaccinated and unvaccinated, to evaluate the variances in knowledge and awareness levels of the disease between the vaccinated and unvaccinated groups. The telephone survey was conducted during the month of February 2005. Survey questions (Table 2) included the identification of risk factors and access to the vaccine. Since this group of patients includes large subpopulations of recent immigrants from Ethiopia and the former Soviet Union, interviewers (employees of a commercial survey firm) fluent in Russian or Amharic in addition to Hebrew conducted the survey. In their opening remarks to the patients, the interviewers stated that the survey was being conducted by the Leumit Health Fund among its patients.

\section{Results}

A total of 672 patients were identified (532 unvaccinated and 140 vaccinated). Vaccination rates were highest among patients who had undergone solid organ transplants (33.3\%), followed by nephrotic syndrome (29.4\%), bone marrow transplants (10.2\%), and HIV (9\%), for an overall rate 20.8\% (Table 3 ).

The interviewers attempted to contact the households or place of work of these patients (21 patients did not have telephones or the telephone number on file was incorrect). Of these, $364(54.2 \%)$ agreed to participate, 87 (12.9\%) refused to participate, and 221 (32.9\%) were not available. The disease distribution and vaccination rates among the surveyed population appear in Table 4. The overall vaccination rate was observed to

\begin{tabular}{l|l}
\hline \multicolumn{1}{c}{ TAB LE 1 ICD-9-CM Diagnosis Codes } \\
\multicolumn{1}{c}{ Used to Identify High-Risk Patients } \\
\hline $\begin{array}{l}\text { ICD-9-CM } \\
\text { Code }\end{array}$ & Description \\
\hline 042 & HIV disease, AIDS \\
33.6 & Combined heart-lung transplantation \\
37.5 & Heart transplantation \\
41.0 & Bone marrow or hematopoietic stem cell transplant \\
41.00 & Bone marrow transplant, not otherwise specified \\
41.01 & Autologous bone marrow transplant without purging \\
41.02 & Allogenic bone marrow transplantation with purging \\
41.03 & Allogenic bone marrow transplantation without purging \\
41.04 & Autologous hematopoietic stem cell transplant without purging \\
41.05 & Allogeneic hematopoietic stem cell transplant without purging \\
41.08 & Allogeneic hematopoietic stem cell transplant with purging \\
41.09 & Autologous bone marrow transplant with purging \\
50.5 & Liver transplant \\
52.8 & Transplantation of pancreas \\
55.6 & Kidney transplantation \\
581 & Nephrotic syndrome \\
581.0 & Nephrotic syndrome with lesion of proliferative glomerulonephritis \\
581.1 & Nephrotic syndrome with lesion of membranous glomerulonephritis \\
581.2 & Nephrotic syndrome with lesion of membranoproliferative \\
& glomerulonephritis \\
581.3 & Nephrotic syndrome with lesion of minimal change glomerulonephritis \\
581.8 & Nephrotic syndrome with other specified pathological lesion in \\
kidney & Nephrotic syndrome in diseases classified elsewhere \\
581.81 & Nephrotic syndrome with unspecified pathological lesion in kidney \\
581.9 & Asymptomatic HIV infection status \\
V08 & Kidney replaced by transplant \\
V42 & Pancreas replaced by transplant \\
V42.01 & Leart replaced by transplant \\
V42.1 & Liver replaced by transplant \\
\hline
\end{tabular}

HIV=human immunodeficiency virus; ICD-9-CM=International Classification of Diseases, Ninth Revision, Clinical Modification.

be higher among the surveyed population than in the target population (26.1\% vs. $20.8 \%$ ). However, this difference was not statistically significant because of the small sample size.

The results of the survey appear in Table 2. Among the nonimmunized patients, $45 \%$ of the respondents replied that they did not know what groups of people are at risk of developing pneumonia; $29.9 \%$ of those who were immunized $(P=0.06)$ responded similarly. Only $15.7 \%$ of the immunized patients claimed that they themselves are not at risk compared with $39.8 \%$ among the nonimmunized respondents $(P<0.001)$. Concerning awareness of the availability of the vaccine, $59.5 \%$ of the immunized patients responded that they were aware of its availability compared with $26.9 \%$ of the nonimmunized $(P<0.001)$. A total of $57.6 \%$ of the immunized patients knew 


\section{TABLE 2}

Patient Questionnaire

(Values Represent \% of Respondents, $\mathrm{n}=364$ )

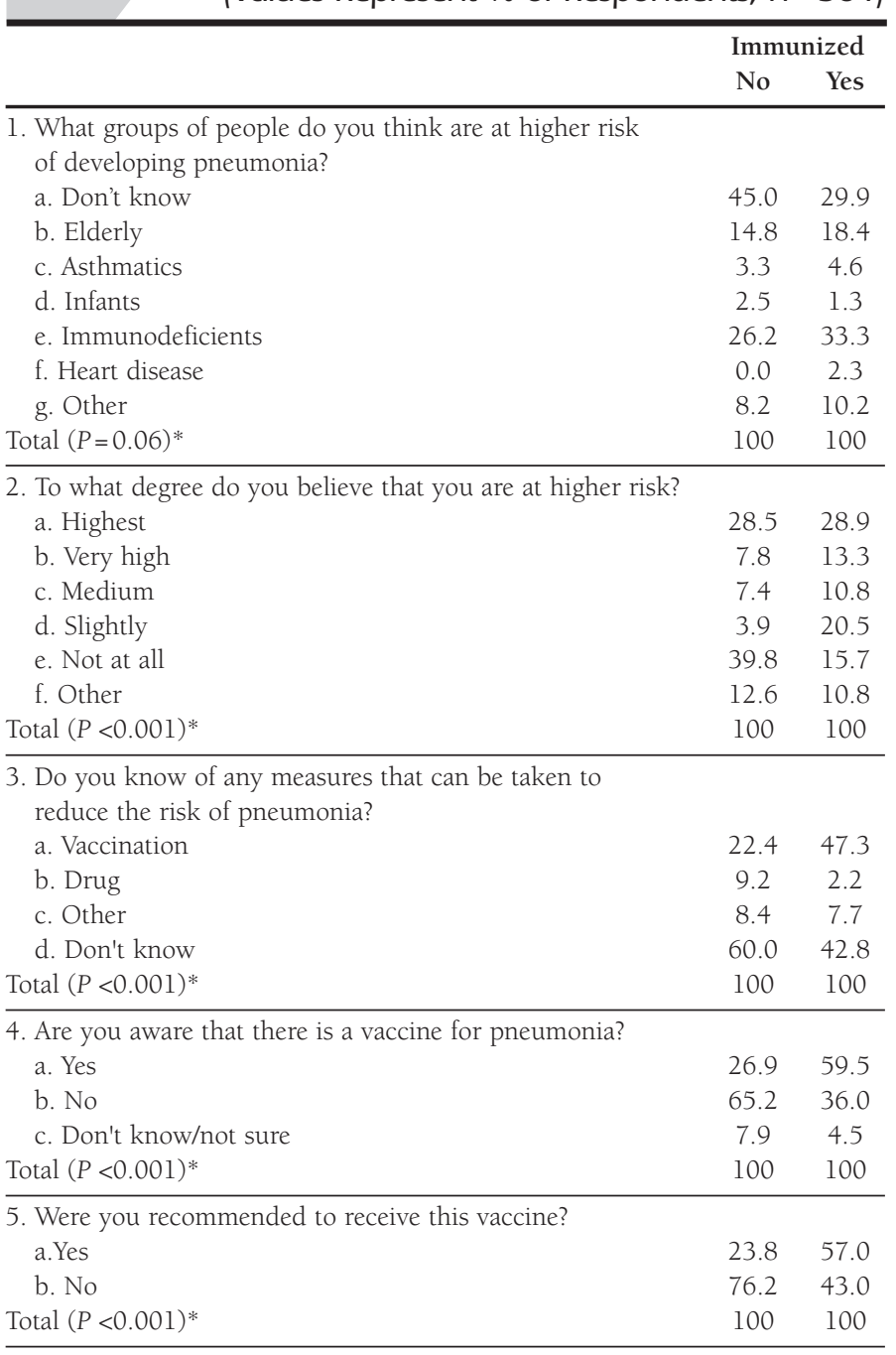

6. Are you aware that you can receive the vaccine free

of charge at your MCO clinic?

$\begin{array}{lll}\text { a. Yes } & 24.7 & 57.6\end{array}$

$\begin{array}{lll}\text { b. No } & 61.5 & 31.5\end{array}$

$\begin{array}{lll}\text { c. Not sure } & 13.8 & 10.9\end{array}$

$\begin{array}{lrr}\text { Total }(P<0.001) * & 100 & 100\end{array}$

\begin{tabular}{lc}
\hline & Nonimmunized $\dagger$ \\
\hline 7. Why did you not receive the vaccine? & \\
a. Unaware of availability & 76.5 \\
b. I am not in a risk group & 5.2 \\
c. Lack of time & 1.4 \\
d. No need due to medical care & 7.5 \\
e. Don't believe in effectiveness of vaccines & 4.7 \\
f. I scheduled an appointment for next month & 0.9 \\
g. Other & 3.8 \\
Total $(P<0.001) *$ & 100 \\
* $P$ values were calculated using the chi-square statistical test. & \\
$\dagger n=269$. & \\
$M C O=$ managed care organization. &
\end{tabular}

that the vaccine is available free of charge compared with $24.7 \%$ of those not immunized $(P<0.01$ ), and $57.0 \%$ of the immunized patients claimed that they were recommended to receive the vaccine compared with $23.8 \%$ among the nonimmunized $(P<0.001)$.

\section{Discussion}

The day after the survey began in February 2005, patients started to appear in Leumit clinics requesting to be immunized. Unfortunately, at that time, stock levels of the vaccine in Israel had been depleted. On the third day of the survey, the public health nurse in charge of the HIV outpatient clinic of one of the country's major medical centers contacted us, stating that, during the past few days, many of the center's Ethiopian immigrant HIV patients had come to the center to tell him that an Amharic-speaking women had called them and asked "all kinds of questions." These patients turned to the health nurse in search of an explanation for what they perceived to be a very unusual and mysterious incident.

We explained the purpose of the study to the nurse and elaborated on the challenges involved in reaching out to this particular group. The nurse suggested that, since these patients come to his clinic on a monthly basis to receive their antiretroviral medications, he could vaccinate the necessary patients if we supplied him with a list of eligible patients and the quantity of vaccines needed. When the vaccine later became available, this procedure was adopted in HIV outpatient clinics throughout Israel. Thus, although this survey was not intended to be a quality improvement intervention per se, it did, in fact, yield favorable QI results.

This study suggests that this MCO must pursue alternative methods for the dissemination of information regarding pneumococcal vaccination that may be vital to the health of particularly vulnerable patients. This point is underscored by the observation of low vaccination rates coupled with the approximately $78 \%$ rate of unvaccinated patients who were unaware of the existence of the vaccine. The serendipitous outcome reported here in which evaluative research generated interest both among patients to receive the vaccine and ancillary care givers to pursue administrative solutions is therefore interesting. These results may indicate that future programs to improve vaccination rates may, in fact, emanate from the awareness created by the initiation of benchmarking and performance measure evaluations in particular patient groups.

It has been suggested that the suboptimal adult immunization rates in the United States may not be attributable to lack of interest among primary care physicians and that most physicians would indeed adopt evidence-based strategies to improve immunization delivery. ${ }^{16}$ Additionally, it has been established that physicianpractice environments that are conducive to patient education and immunization-promotion activity do achieve higher vaccination rates. ${ }^{17}$ Accordingly, improved vaccination rates may be achieved 
in the future through multifaceted programs composed of evidence-based delivery strategies implemented in conjunction with organizational changes that create practice environments more suitable for promotion of vaccination. Similarly, administrative and/or financial support may be necessary in some settings to change current policy environments. ${ }^{18}$ Likewise, the recent failure of a media campaign in the United States to promote pneumococcal vaccination ${ }^{19}$ reinforces this premise that future efforts should be targeted to the clinical practice setting. The results presented here suggest additional approaches to inducing compliance with vaccination programs.

The present study raises the issue of expanding the HEDIS performance measures to include vaccination rates among immunocompromised patients. Such a measure could constitute a benchmark that MCOs could use to improve pneumococcal vaccination rates among this vulnerable population. The general paucity of studies evaluating factors associated with suboptimal vaccination rates in this population may have contributed to the lack of attention paid by policy makers to this issue. Alternatively, financial considerations or a belief that a vaccine policy would be too difficult to administer may have impeded the inclusion of relevant performance measures. In this context, HEDIS performance measures could be utilized in the future as evaluation tools of processes in which evidence-based guideline formulation and propagation and environmental changes coalesce into an integrative QI program.

The success of the survey itself in generating interest in the community among individual patients and then, consequentially, among other health care providers may provide insight into potential avenues with which to approach this challenge. The postcards sent to high-risk health plan members had small apparent impact on the pneumococcal vaccination rates among high-risk patients. Direct contact with the patients, with or without a survey, appears to be necessary, particularly among more insular immigrant populations from the developing world, such as the Ethiopians in this study, who must cope with the complexities of modern Western medical care that are nonexistent in their country of origin..$^{20}$

In addition to telephone contact, case mangers may need to assume responsibility for pneumococcal vaccination of highrisk patients. Since many of these patients receive chronic care in non-MCO facilities, there will be challenges in coordinating care among a multitude of providers, making intervention by the case manager particularly important. Lastly, we learned from the public health nurse that some solutions might be easily implemented through cooperation with junior-level staff of non-MCO facilities.

\section{Conclusion}

The pneumococcal vaccination rates among immunocompromised patients in this MCO in 2005 were found to be inadequate. A more effective approach, such as direct contact with the

\begin{tabular}{|c|c|c|c|}
\hline \multirow[b]{2}{*}{ Diagnosis } & \multicolumn{2}{|c|}{ No. of Patients (\%) } & \multirow[b]{2}{*}{ Total (\%) } \\
\hline & Nonimmunized & Immunized & \\
\hline Bone marrow transplant & $62(89.9)$ & $7(10.2)$ & $69(10.3)$ \\
\hline HIV & $242(91.0)$ & $24(9.0)$ & $266(39.6)$ \\
\hline Nephrotic syndrome & $60(70.6)$ & $25(29.4)$ & $85(12.6)$ \\
\hline Solid organ transplant & $168(66.7)$ & $84(33.3)$ & $252(37.5)$ \\
\hline Total & $532(79.2)$ & $140(20.8)$ & $672(100)$ \\
\hline
\end{tabular}

\begin{tabular}{|c|c|c|c|}
\hline \multirow[b]{2}{*}{ Diagnosis } & \multicolumn{2}{|c|}{ No. of Patients (\%) } & \multirow[b]{2}{*}{ Total (\%) } \\
\hline & Nonimmunized & Immunized [95\% CI] & \\
\hline Bone marrow transplant & $31(88.6)$ & 4 (11.4) [0.3-22.5] & $35(9.6)$ \\
\hline HIV & $99(86.8)$ & 15 (13.2) [6.9-19.5] & $114(31.3)$ \\
\hline Nephrotic syndrome & $40(71.4)$ & 16 (28.6) [16.4-40.8] & $56(15.4)$ \\
\hline Solid organ transplant & $99(62.3)$ & $60(37.7)[30.1-45.3]$ & $159(43.7)$ \\
\hline Total/average & $269(73.9)$ & $95(26.1)[21.7-30.6]$ & $364(100)$ \\
\hline
\end{tabular}

patient through a case manager, is necessary to increase vaccination rates. It will be necessary to pay particular attention to insular immigrant populations, especially among those who are unfamiliar with Western medical care. Evaluation of vaccination programs based on member surveys has emerged as a potentially effective tool in this respect, which may be considered by health plans in other countries such as the United States.

\section{DISCLOSURES}

Partial funding for this research was provided by MSD Pharmaceuticals Israel, Petach Tikvah, and was obtained by Leumit Health Fund. The authors do not provide consulting or other compensated services for MSD; they disclose no potential bias or conflict of interest relating to this article. Natan R. Kahan served as principal author of the study. Study concept and design were contributed by Kahan and authors Dan-Andrei Waitman, Shimon Blackman, and David P. Chinitz. Data collection was primarily the work of Kahan and Blackman, with input from Waitman; data interpretation was the work of Kahan, Waitman, and Chinitz. Drafting of the manuscript and its critical revision was primarily the work of Kahan, with input from the coauthors.

\section{REFERENCES}

1. Siriwardena AN. Targeting pneumococcal vaccination to high-risk groups: a feasibility study in one general practice. Postgrad Med J. 1999;75:208-12.

2. Pierce AB, Hoy JF. Is the recommendation for pneumococcal vaccination of HIV patients evidence based? J Clin Virol. 2001;22:255-61. 
3. Overturf GD. American Academy of Pediatrics. Committee on Infectious Diseases. Technical report: prevention of pneumococcal infections, including the use of pneumococcal conjugate and polysaccharide vaccines and antibiotic prophylaxis. Pediatrics. 2000;106:367-76.

4. Centers for Disease Control and Prevention. Recommendations of the Advisory Committee on Immunization Practices (ACIP): use of vaccines and immune globulins in persons with altered immunocompetence. MMWR Morb Mortal Wkly Rep. 1993;42(RR-04):1-18.

5. Ramachandra J, Bond A, Ranaboldo C, Cullis J. An audit of post-splenectomy prophylaxis—are we following the guidelines? Ann R Coll Surg Engl. 2003;85: 252-55.

6. Brigden ML, Pattulo A, Brown G. Pneumococcal vaccine administration association with splenectomy: the need for improved education, documentation, and the use of a practical checklist. Am J Hematol. 2000;65:25-29.

7. Duchini A, Gross JA, Karpen S, Pockros PJ. Vaccination for adult solidorgan transplant recipients: current recommendations and protocols. Clin Microbiol Rev. 2003;16:357-64.

8. Ballout A, Goffin E, Yombi JC, Vandercam B. Vaccinations for adult solid organ transplant recipient: current recommendations. Transplant Proc. 2005;37:2826-27.

9. Ontario Ministry of Health and Long-Term Care. Immunization: pneumococcal vaccine (polysaccharide-for age 2 years and over). Available at: http://www.health.gov.on.ca/english/public/pub/immun/pnem.html. Accessed November 25, 2005.

10. Centers for Disease Control and Prevention. Prevention of pneumococcal disease: recommendations of the Advisory Committee on Immunization Practices (ACIP). MMWR Morb Mortal Wkly Rep. 1997;46(RR-08):1-24.

11. Shapiro ED, Berg A, Austrian R, et al. The protective efficacy of polyvalent pneumococcal polysaccharide vaccine. N Engl J Med. 1991;325:1453-60.
12. Centers for Disease Control and Prevention. Improving influenza, pneumococcal polysacharide, and hepatitis B vaccination coverage among adults aged $<65$ years at high risk: a report of the Task Force on Community Preventive Services. MMWR Morb Mortal Wkly Rep. 2005;54(RR-5). Available at: http://www.cdc.gov/mmwr/preview/mmwrhtml/mm5343a2.htm. Accessed December 31, 2005 .

13. Draft HEDIS. 2001. Available at: http://ncqa.org/docs/drafts/ h2kldraft.doc. Accessed January 4, 2006.

14. NQF-endorsed national voluntary consensus standards for physicianfocused ambulatory care: NCQA measures. October 2005. Vol. 3. Available at: http://www.ncqa.org/main/nqf_posting_table.pdf. Accessed January 4, 2006.

15. HEDIS 2006 summary table of measures and product lines. Available at: http://www.ncqa.org/Programs/HEDIS/HEDIS_2006_Summary.pdf. Accessed January 4, 2006.

16. Szilagyi PG, Shone LP, Barth R, et al. Physician practices and attitudes regarding adult immunizations. Prev Med. 2005;40:152-61.

17. Nowalk MP, Bardella IJ, Zimmerman RK, Shen S. The physician's office: can it influence adult immunization rates? Am J Manag Care. 2004;10:13-19.

18. Goldstein AO, Kincade JE, Resnick JE, Gamble G, Bearman RS. Policies to increase influenza and pneumococcal immunizations in chronically ill and institiutionalized settings. Am J Infect Control. 2005;33:463-68.

19. Holzman GS, Harwell TS, Johnson EA, Goldbaum, Helgerson SD. A media campaign to rpomote pneumococcal vaccinations: Is a telephone survey an effective evaluation strategy? J Public Health Manag Prac. 2005;11:228-34.

20. Reiff M, Weingarten MA. Illness and treatment perceptions of Ethiopian immigrants and their doctors in Israel. Am J Public Health. 1999;89:1814-18. 